\title{
ESTIMATIVAS DAS PROPRIEDADES DE COMPOSTOS LVL PRODUZIDOS COM PARICÁ (Schizolobium amazonicum Huber ex Ducke) POR MEIO DE STRESS WAVE ${ }^{1}$
}

\begin{abstract}
Rafael Rodolfo Melo² e Cláudio Henrique Soares Del Menezzi ${ }^{3}$
RESUMO - Foram avaliadas as propriedades físicas e mecânicas de compósitos LVL produzidos com a madeira de paricá (Schizolobium amazonicum Huber ex. Ducke), por meio de Stress Wave Timer. Os compósitos foram confeccionados em laboratório, dos quais foram retiradas amostras, que foram inicialmente destinadas à realização dos ensaios não destrutivos. Todas as amostras, com dimensões de 2,2 $\times 2,2 \times 40 \mathrm{~cm}$, foram ensaiadas, não destrutivamente, com a propagação de ondas nos sentidos flatwise e edgewise. Em sequência, as mesmas amostras foram destinadas à confecção de subamostras, para realização dos ensaios destrutivos, físicos (absorção de água, inchamento em espessura e inchamento residual) e mecânicos (resistência e rigidez à flexão estática flatwise; resistência e rigidez à flexão estática edgewise; resistência à compressão paralela e resistência ao cisalhamento paralelo e perpendicular). A velocidade de propagação das ondas (V0) e o módulo de elasticidade dinâmico $\left(E_{m d}\right)$, obtidos com o auxílio do Stress Wave, foram utilizados para elaboração de modelos de predição das propriedades avaliadas. Os resultados indicaram que o Stress Wave Timer apresenta resultados satisfatórios para predição das propriedades mecânicas de compósitos LVL. Com relação às propriedades físicas, embora tenham sido verificados modelos com ajustes significativos, constatou-se limitação dessa ferramenta para predição desses parâmetros. Contudo, considerando ambas as propriedades, físicas e mecânicas, os melhores ajustes foram observados em amostras ensaiadas com a propagação de ondas no sentido edgewise e com o uso da variável independente $E_{m d}$.
\end{abstract}

Palavras-chave: Ondas de tensão; Propriedades físico-mecânicas; Compósitos estruturais de madeira.

\section{PREDICTION OF THE PROPERTIES OFLVL PRODUCED WITH PARICÁ (Schizolobium amazonicum Huber exDucke) BYMEANS OF STRESS WAVE METHOD}

\begin{abstract}
The physical and mechanical properties of the LVL manufactured with paricá (Schizolobium amazonicum Huber ex. Ducke) was evaluated by Stress Wave Timer. The LVL composite was made in laboratory and from them some samples were withdrawn, that were initially destined for the non-destructive tests. All samples with $2.2 \times 2.2 \times 40$ cmwere tested, non-destructively, in the wave propagation direction flatwise and edgewise. Afterwards, the same samples were transformed into subsamples, to be used in destructive tests: physical (water absorption; thickness swelling and residual swelling) and mechanical tests (resistance and rigidity to flatwise static bending; resistance and rigidity to edgewise static bending; resistance to parallel compression and resistance toparallel and perpendicular shearing). The propagation wave velocity $\left(V_{0}\right)$ and the dynamic elasticity modulus $\left(E_{m d}\right)$, obtained with the support of Stress Wave, were used for modeling the properties. The results showed that the Stress Wave Timer has satisfactory predictions for mechanical properties of LVL. As for the physical properties, although some models had significant settings, it was found limitation of this tool for the prediction of these parameters. However, in bothphysical and mechanicalproperties, the best settings were observed in samples tested with the wave propagation in edgewise direction, using the $E_{m}$ dindependent variable.
\end{abstract}

Keywords: Stress wave; Physical-mechanical properties; Structural composite lumber.

\footnotetext{
${ }^{1}$ Recebido em 07.05.2014 aceito para publicação em 02.10.2014.

${ }^{2}$ Universidade Federal de Mato Grosso, Instituto de Ciências Agrárias e Ambientais, MT, Brasil. E-mail: <rrmelo2@yahoo.com.br>.

${ }^{3}$ Universidade de Brasília, Faculdade de Tecnologia, Departamento de Engenharia Florestal, Brasília, DF, Brasil. E-mail: <cmenezzi@unb.br>.
} 


\section{INTRODUÇÃO}

No Stress Wave Timer, as ondas são geradas pela vibração produzida por um impacto na peça em estudo. A propagação da onda é induzida por meio de pêndulo ou martelo. Ao ser acionada, a onda em um acelerômetro de partida inicia-se uma cronometragem de tempo, a qual é encerrada quando a onda atinge o acelerômetro de parada. O método utiliza baixos movimentos moleculares de tensão para medir duas propriedades fundamentais dos materiais: a energia armazenada e a dissipação. A primeira manifesta-se pela velocidade com a qual a onda percorre o material, enquanto a taxa sob a qual a onda é atenuada é uma indicação de dissipação de energia (MORALES et al., 2013).

Souza (2009) destacou que a onda emitida pelo Stress Wave pode ser excelente indicador das propriedades mecânicas, tanto de madeiras quanto dos produtos à base de madeira. Segundo esse autor, os parâmetros obtidos nos ensaios com ondas de tensão- velocidade de propagação $\left(V_{0}\right)$ e o módulo de elasticidade dinâmico $\left(E_{m d}\right)$ podem também proporcionar resultados satisfatórios para predição das propriedades de compostos laminados, aglomerados e chapas de fibras, entre outros.

Vários pesquisadores têm estudado o uso da técnica das ondas acústicas na estimativa das propriedades de compostos LVL por meio do Stress Wave (JUNG, 1982; CHA; PEARSON, 1994; MATOS, 1997; BORTOLETTO JÚNIOR, 2006; GABRIEL, 2007; SOUZA et al., 2011; MELO; DEL MENEZZI, 2013; MELO et al., 2014). Os resultados têm demonstrado a existência de elevadas correlações entre a determinação do módulo de elasticidade mecânico e o módulo de elasticidade estimado, por meio de ondas acústicas. Além disso, Matos (1997) destacou que a montagem dos LVL, utilizando lâminas previamente classificadas e agrupadas por classe de qualidade, tem demonstrado muita eficiência, conferindo elevadas propriedades mecânicas finais aos LVL.

Apesar das inúmeras evidências de eficácia do Stress Wave para predição das propriedades dos compostos de madeira, a maioria dos trabalhos tem-se concentrado quase que, exclusivamente, no uso dessa ferramenta para predição das propriedades mecânicas de resistência e rigidez da madeira e dos produtos de madeira. Em contrapartida, poucos estudos têm sido realizados objetivando à utilização dessas ferramentas, na predição de parâmentos relacionados às propriedades físicas da madeira e dos produtos de madeira, principalmente com relação aos parâmetros da estabilidade dimensional (inchamento em espessura, absorção de água e taxa de não retorno em espessura).

Este trabalho tem como objetivo estimar as propriedades físicas e mecâminas de compostos LVL produzidos com paricá (Schizolobium amazonicum Huber ex. Ducke), por meio da utilização da técnica de ondas de tensão (Stress Wave).

\section{MATERIAL E MÉTODOS}

\subsection{Obtenção das lâminas e manufatura dos LVL}

Foram utilizadas lâminas de paricá (Schizolobium amazonicum) de aproximadamente $0,27 \mathrm{~cm}$ de espessura, obtidas em laminadoras do Município de Dom Eliseu, Estado do Pará. Essas lâminas foram selecionadas e redimensionadas para confecção dos LVL. A resina empregada foi a fenol-formaldeído (CR-7010), na gramatura de $200 \mathrm{~g} / \mathrm{m}^{2}$, com viscosidade de $840 \mathrm{cP}$ e teor de sólidos de $59 \%$.

Após a aplicação do adesivo, foi realizada uma pré-prensagem, a frio, dos LVLdurante $30 \mathrm{~min}$. Em seguida, estes foram levados à prensa hidráulica e submetidos à prensagem por $12 \mathrm{~min}$, com pratos aquecidos a 140 ${ }^{\circ} \mathrm{C}$ e pressão de $1,0 \mathrm{~N} / \mathrm{mm}^{2}$. Ao todo, foram produzidos 21 compostos laminados com dimensões aproximadas de $25 \mathrm{~cm} \times 2,2 \mathrm{~cm} \times 50 \mathrm{~cm}$ (largura $\times$ espessura $\times$ comprimento).

\subsection{Realização dos ensaios não destrutivos}

Depois de climatizados, os LVL foram esquadrejados e, novamente, conduzidos ao laboratório para serem tomadas suas massas e dimensões, as quais foram utilizadas no cálculo da massa específica. Na mesma ocasião, foi determinado o tempo de propagação da onda em cada painel, utilizando o Stress Wave Timer. Posteriormente, esse valor foi utilizado para determinação da velocidade de propagação de ondas $\left(V_{0}\right)$ e para o cálculo do módulo de elasticidade dinâmico $\left(E_{m d}\right)$ (equações 1 e 2). Após os ensaios não destrutivos, os compostos retornaram à marcenaria para retiradas das amostras destinadas à realização dos ensaios físicos e mecânicos.

$V_{0}=\frac{L}{t \times 10^{-6}}$

Revista Árvore, Viçosa-MG, v.38, n.6, p.1155-1163, 2014 
$E_{m d}=\frac{V_{0} \times \rho}{g} \times 10^{-5}$

em que $V_{0}=$ velocidade de propagação da onda, $\mathrm{m} / \mathrm{s} ; E_{m d}=$ módulo de elasticidade dinâmico, $\mathrm{N} / \mathrm{mm}^{2}$; $L=$ distância percorrida pela onda, $\mathrm{m} ; t=$ tempo de trânsito da onda, $\mu \mathrm{s} ; \tilde{n}=$ massa específica aparente, $\mathrm{kg} / \mathrm{m}^{3}$; e $g=$ aceleração da gravidade, $9,804 \mathrm{~m} / \mathrm{s}^{2}$.

\subsection{Determinação das propriedades}

$\mathrm{Na}$ avaliação das propriedades físicas (massa específica, teor de umidade, absorção de água, inchamento em espessura e inchamento residual) dos LVL produzidos com paricá, foram utilizadas recomendações da Norma ASTM D 1037 (1999). Foram utilizadas 21 amostras de madeira por tratamento, nas dimensões de $2,2 \mathrm{~cm}$ $\times 2,2 \mathrm{~cm} \times 10 \mathrm{~cm}$. As dimensões e massa dos corpos de prova foram tomadas após a climatização. Em seguida, foram submersos em água, durante $96 \mathrm{~h}$, quando tiveram suas dimensões e massas retomadas. Ao término do ensaio, as amostras foram novamente conduzidas à câmara climática e, após sua estabilização, tiveram suas dimensões e massa obtidas novamente.

Os ensaios mecânicos foram conduzidos conforme recomendações da Norma ASTM D 5456 (2006). Para o ensaio de resistência à flexão, avaliaram-se as posições flatwise e edgewise (Figura 1). Foram utilizadas 21 amostras/tratamento/posição, nas dimensões de 2,2 $\mathrm{cm} \times 2,2 \mathrm{~cm} \times 40 \mathrm{~cm}$. A velocidade de aplicação de carga foi de aproximadamente $2,5 \mathrm{~mm} / \mathrm{min}$, para um vão de $36 \mathrm{~cm}$ de comprimento.

Na resistência à compressão, utilizaram-se 21 amostras por tratamento, com dimensões de $2,2 \mathrm{~cm}$ $\times 2,2 \mathrm{~cm} \times 10 \mathrm{~cm}$. As dimensões dos corpos de prova foram determinadas pela máxima relação comprimento/ raio de giração (17 vezes) permitida pela norma. Com a velocidade do ensaio de $1 \mathrm{~mm} / \mathrm{min}$, determinou-se a resistência à compressão das amostras, pela relação entre a força aplicada e a área sujeita ao esforço. Para o cisalhamento paralelo e perpendicular à linha de cola, foram avaliadas 21 amostras com dimensões de 2,2 $\mathrm{cm} \times 2,2 \mathrm{~cm} \times 3,3 \mathrm{~cm}$ e um área destinada à aplicação do esforço cisalhante.

\subsection{Análise dos resultados}

Buscou-se o ajuste de equações lineares simples que visavam estimar o desempenho dos LVL quanto às suas propriedades físicas (absorção de água; inchamento em espessura; e inchamento residual) e mecânicas (resistência e rigidez à flexão estática flatwise; resistência e rigidez à flexão estática edgewise; resistência à compressão paralela; e resistência ao cisalhamento paralelo e perpendicular), observadas nos ensaios não destrutivos, em função da velocidade de propagação das ondas e do módulo de elasticidade dinâmico, obtidos

Fonte: Adaptado de SOUZA, 2009 Source:Adaptedfrom SOUZA, 2009.
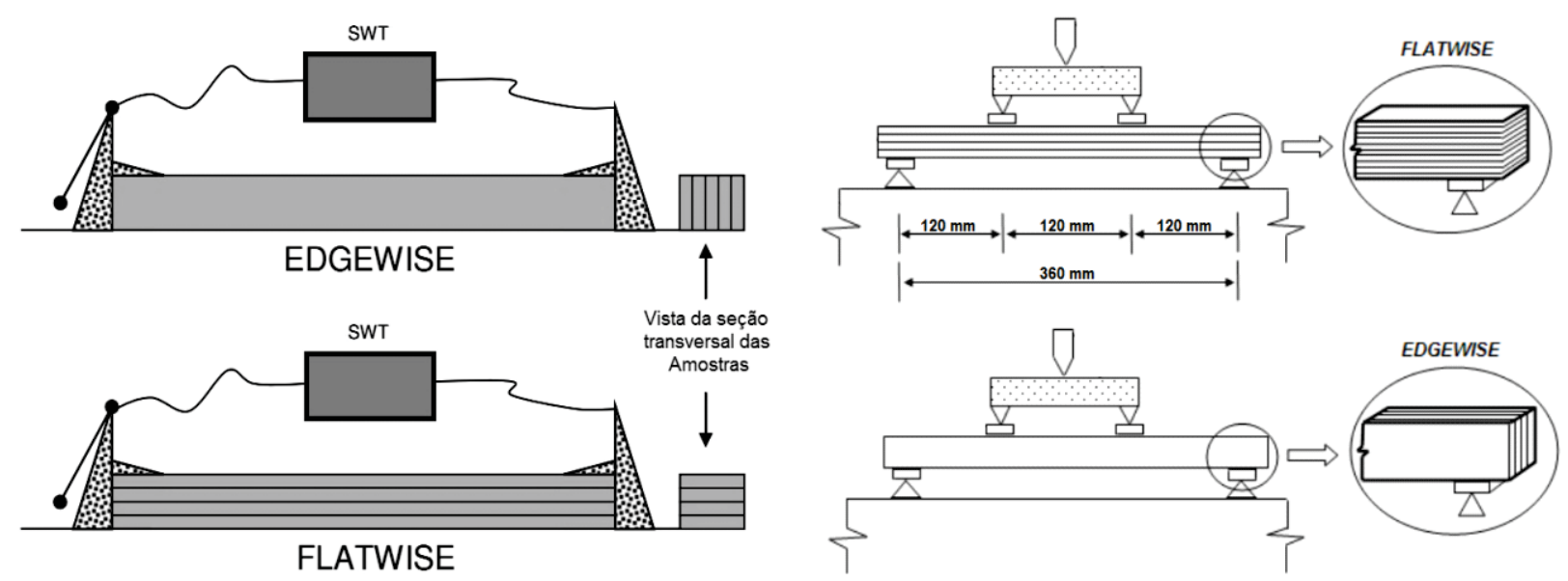

Figura 1 - Procedimentos realizados nos ensaios não destrutivos com Stress Wave Timer (SWT) e destrutivos das amostras submetidas a ensaios de flexão estática.

Figure 1 - Procedures performed in non-destructive tests with Stress Wave Timer (SWT) and destructive test for samples subject to static bending tests. 
por Stress Wave (com propagação de ondas no sentido flatwise e stress wave, com propagação de ondas no sentido edgewise). Também, foi estimada a relação entre o $E_{m d}$, observado nos compostos LVL, e a média das amostras retiradas de capa painel.

\section{RESULTADOS}

Na Figura 2 podem ser observadas as relações entre o módulo de elasticidade dinâmico $\left(E_{m d}\right)$, obtido para os compostos LVL, e os valores médios verificados para os corpos de prova utilizando Stress Wave.

A estimativa dos parâmetros físicos dos LVL de paricá, utilizando Stress Wave, podem ser observados na Figura 3 (absorção de água e inchamento em espessura). Verifica-se que o aumento do módulo de elasticidade dinâmico contribui, significativamente, para redução na absorção de água. Já para o inchamento em espessura ocorre comportamento inverso, ou seja, o inchamento em espessura dos LVL apresenta-se diretamente proporcional ao módulo de elasticidade dinâmico. Não foi possível a elaboração de modelos significativos para predição do inchamento residual, também conhecido como taxa de não retorno em espessura.

O ajuste de equações, para estimativa das propriedades mecânicas, pode ser observado nas Figuras 4 (resistência à flexão flatwise (A e B) e resistência à flexão edgewise $(\mathrm{C}$ e D)) e Figura 5 (resistência à compressão paralela). Em todos esses casos, verificou-se que o aumento do módulo de elasticidade dinâmico incrementou a resistência dos compostos LVL. Nos ensaios de resistência ao cisalhamento, paralelo e perpendicular, não foi possível a elaboração de modelos com ajustes significativos estatisticamente, considerando $\alpha=0,05$.

\section{DISCUSSÃO}

\subsection{Ensaios não destrutivos dos compostos LVL $x$ Ensaios não destrutivos das amostras}

A relação entre os valores do módulo de elasticidade dinâmico $\left(E_{m d}\right)$ dos painéis versus o valor médio das amostras obtidas desses indica que, mesmo em compostos laminados confeccionados em laboratório, com matériaprima selecionada e condições de produção controladas, pode haver significativas variações dentro de um mesmo painel (Figura 2). Segundo Trevisan et al. (2008), essas variações podem estar relacionadas a diversos aspectos, sendo a principal delas as características intrínsecas da própria natureza da madeira, que apresenta variações consideráveis em suas propriedades, tanto no sentido base-topo quanto no sentido medula-casca.

Ainda na Figura 2, observa-se que, apesar de a equação gerada ser estatisticamente significativa a $99 \%$ de confiança, o valor observado para o coeficiente de determinação $\left(\mathrm{R}^{2}=0,57\right)$ foi menor do que o comumente observado na literatura, o que indica variabilidade ao longo da superfície de cada painel. Gabriel (2007), em análise semelhante, observou coeficientes de determinação $\mathrm{R}^{2}$ superiores a 0,80 para o mesmo parâmetro.

\subsection{Propriedades físicas}

Embora diversas pesquisas venham demonstrando a eficácia dos métodos não destrutivos, na estimativa das propriedades mecânicas dos LVL (MATOS, 1997; BORTOLETTO JÚNIOR, 2006; GABRIEL, 2007; ROSS et al., 2004; VALLANCE, 2009), poucos estudos têm sido desenvolvidos, visando à utilização dessas ferramentas, na predição de parâmentos relacionados à estabilidade dimensional desses produtos. Neste estudo, foi possível estimar, de forma significativa, os percentuais de absorção de água e inchamento em espessura dos LVL. Em ambos os casos, a propagação de ondas no sentido edgewise, conjuntamente com a variável independente $E_{m d}$, foram as variáveis que apresentaram a maior eficiência para predição dos parâmetros físicos (Figura 3).

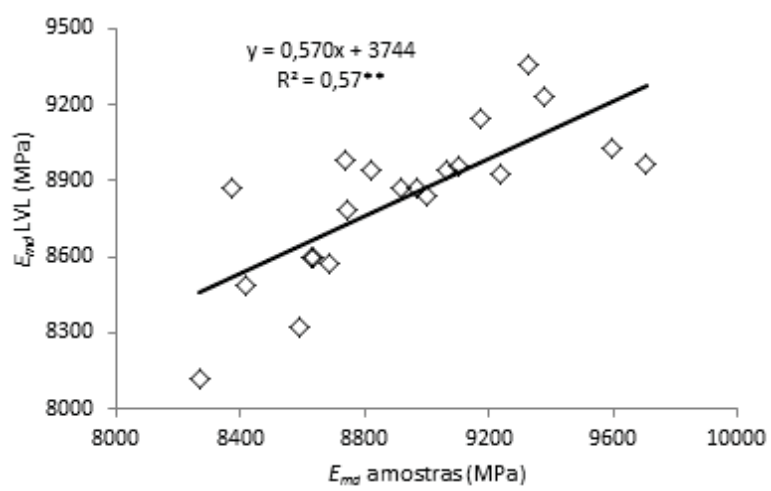

Figura 2 - Relação entre o módulo de elasticidade dinâmico $\left(E_{m d}\right)$ observado nos compostos LVL e nas amostras utilizando Stress Wave.**Significativoa 0,01 .

Figure 2 - Relation between the dynamic elasticity modulus $\left(E_{\text {do }}\right)$ observed in $L V L$ and the samples using Stress Wave. **Significanceat 0.01 . 
$\mathrm{Na}$ absorção de água foi observada tendência de redução com o aumento do $E_{m d}$, enquanto no inchamento em espessura ocorreu o inverso. Esse comportamento pode ser atribuído ao fato de que em madeiras, ou compostos de madeiras, as variáveis $E_{m d}$ são diretamente propocionais à sua massa específica (PELLERIN; ROSS, 2002). Desse modo, conforme constatado por Melo e Del Menezzi (2010), a maior absorção de água observada nas amostras com menor $E_{m d}$ pode ser atribuída ao maior volume de espaços vazios dessas amostras, que possam ser ocupados por água. No entanto, para o inchamento em espessura, amostras mais densas têm maior material e, assim, a contribuição do inchamento
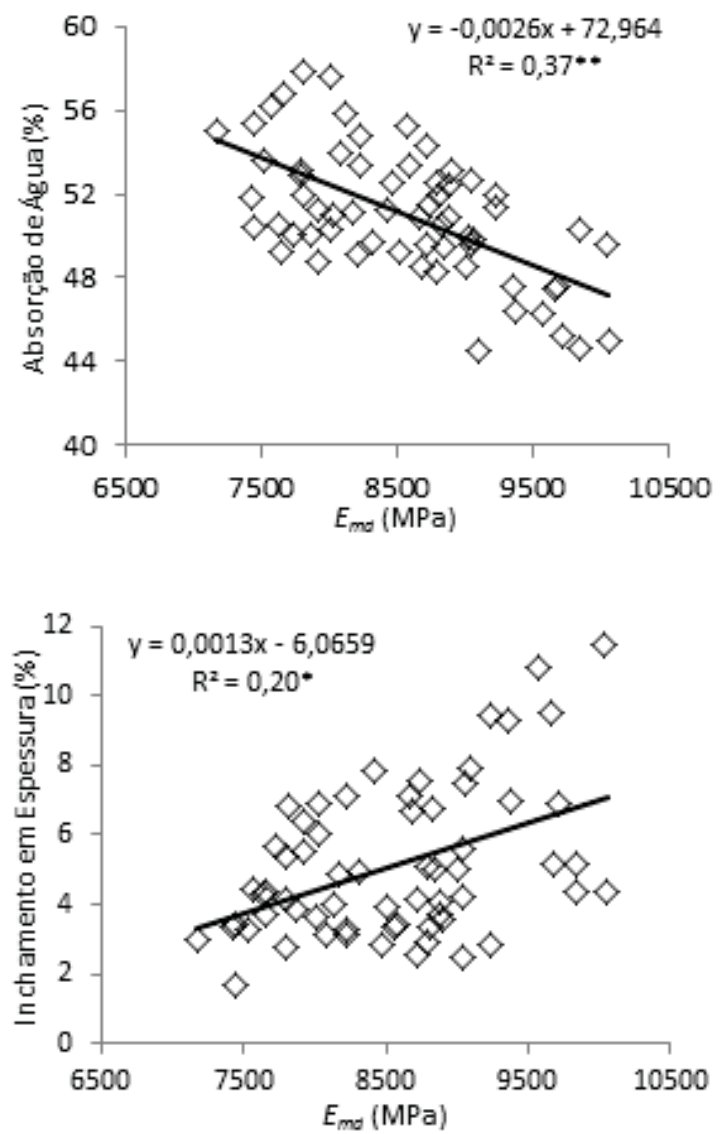

Figura 3 - Estimativa da absorção água (A) e inchamento em espessura (B), em função do módulo de elasticidade dinâmico $\left(E_{m d}\right)$ obtido por meio do Stress Wave. ${ }^{* *}$ Significativoa 0,$01 ; \mathrm{e} *$ Significativoa 0,05 .

Figure 3 - Prediction of water absorption (A) and thickness swelling (B) as a function of the dynamic elasticity modulus $\left(E_{m d}\right)$ obtained by the Stress Wave. ${ }^{* *}$ Significanceat 0.01 ; and ${ }^{*}$ Significanceat 0.05 . higroscópico é maior. Entretanto, Suchsland (1973) destacou que essa relação não é bem defenida e que, diferentemente do que ocorre com a madeira sólida, em que peças mais densas geralmente apresentam inchamento superior; em compostos de madeira, isso não acontece, necessariamente.

Não foi possível predizer, de forma significativa, o inchamento residual observado nos compostos LVL. Esse resultado pode ser atríbuido por ser essa propriedade um parâmetro decorrente, principalmente, da liberação das tensões de compressão (DEL MENEZZI, 2006), tendo a composição física do material menor influência (propriedades morfológicas e anatômicas), que é o principal aspecto diagnosticado pelos métodos não destrutivos (SHIMOYAMA, 2005).

\subsection{Propriedades mecânicas}

O ajuste de equações das amostras submetidas à flexão estática, na posição flatwise, apontou que o $E_{m d}$ com propagação de ondas, no sentido edgewise, pode ser utilizado na predição da resistência e rigidez dos LVL produzidos com paricá (Figura 4). Resultados semelhantes foram observados por Xue e $\mathrm{Bu}$ (2010) e Almeida (2011), ao avaliarem a utilização de diferentes métodos de avaliação não destrutiva. Entre os métodos empregados, o uso do stress wave, com propagação de ondas no sentido edgewise, foi o que proporcionou a definição de modelos com os melhores ajustes, tanto para $\mathrm{E}_{\mathrm{m}}$ quanto para $f_{m}$.

Na comparação entre os ajustes das equações para o módulo de elasticidade $\left(E_{m}\right)$ e módulo de ruptura $\left(f_{m}\right)$ em flexão estática, no sentido flatwise foram observados os melhores ajustes para $E_{m}$. Segundo Stangerlin et al. (2011), esse comportamento se deve ao fato de que a tensão induzida durante os ensaios dinâmicos é pequena, ou seja, as medições dinâmicas são baseadas nas propriedades mecânicas, apenas no limite elástico. $\mathrm{O}$ módulo de ruptura acontece em maior tensão e depois do limite elástico, resultando, consequentemente, em predições menos eficazes, por meio dos ensaios não destrutivos. Grabiel (2007), Santos (2008) e Souza (2009) também observaram esse comportamento em compostos LVL produzidos com lâminas de diferentes espécies florestais.

Neste estudo, não foi possível estimar a resistência e a rigidez à flexão por meio da velocidade de propagação de ondas $\left(V_{0}\right)$. O uso desse parâmetro, como variável 
A
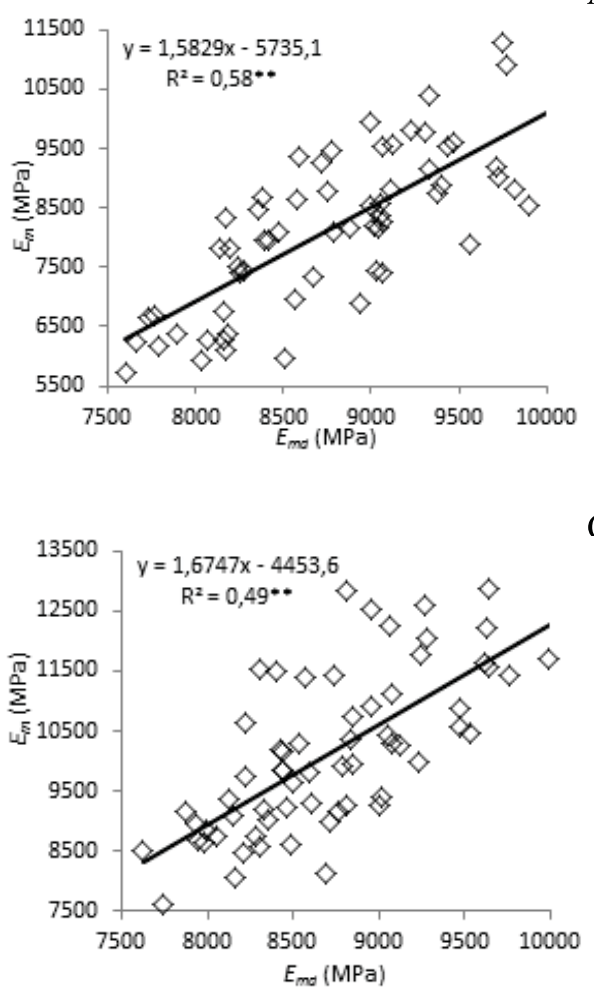

$\mathrm{C}$
B
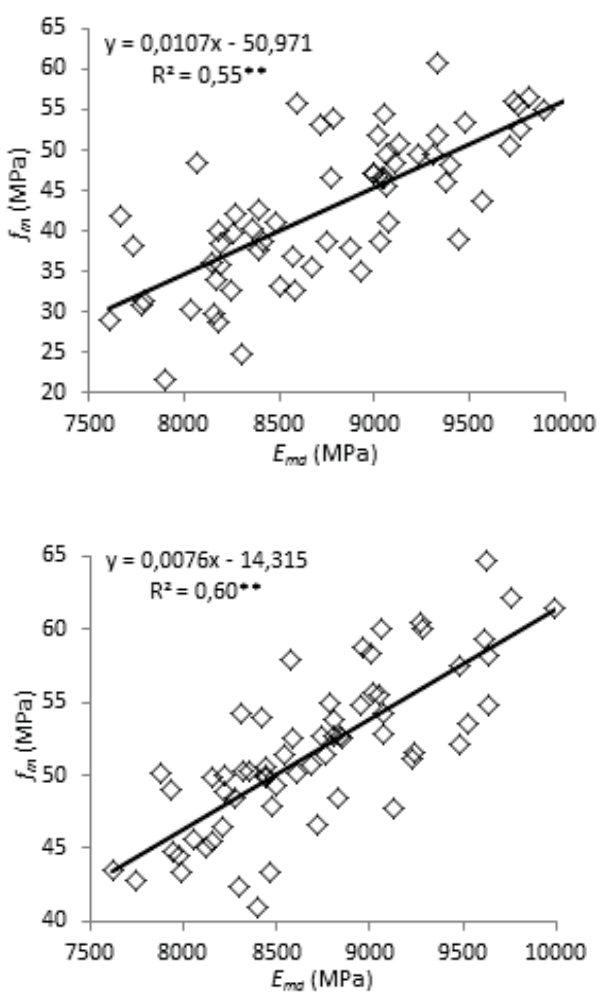

$\mathrm{D}$

Figura 4 - Estimativa dos módulos de elasticidade $\left(\mathrm{E}_{\mathrm{m}}\right)$ e ruptura $\left(\mathrm{f}_{\mathrm{m}}\right)$ na posição flatwise, em função do módulo de elasticidade dinâmico $\left(\mathrm{E}_{\mathrm{md}}\right)$ obtido por meio de stress wave, com propagação no sentido edgewise (A e B); Estimativa dos módulos de elasticidade $\left(\mathrm{E}_{\mathrm{m}}\right)$ e ruptura $\left(\mathrm{f}_{\mathrm{m}}\right)$ na posição edgewise, em função do módulo de elasticidade dinâmico $\left(\mathrm{E}_{\mathrm{md}}\right)$ obtido por meio de stress wave, com propagação no sentido edgewise $(\mathrm{C}$ e D).**Significativoa 0,01 .

Figure 4 - Prediction of the elasticity modulus $\left(E_{m}\right)$ and rupture $\left(f_{m}\right)$ in flatwise position as a function of the dynamic elasticity modulus $\left(E_{m d}\right)$ obtained by the stress wave propagating in the edgewise direction (A and $\left.B\right)$; Prediction of the elasticity modulus $\left(E_{m}\right)$ and rupture $\left(f_{m}\right)$ in edgewiseposition as a function of the dynamic elasticity modulus $\left(E_{m d}\right)$ obtained by the stress wave propagating in the edgewise direction (C and D). ${ }^{* * S i g n i f i c a n c e ~ a t ~ 0.01 . ~}$

independente, não proporciou o ajuste de modelos significativos para predição dos módulos de elasticidade e ruptura, indicando ser a velocidade de propagação de ondas, isoladamente, inadequada para estimar as propriedas LVL produzidas com paricá em ensaios de flexão estática. Todavia, alguns autores, como Halabe et al. (1997) e Souza et al. (2011), apontaram a potencialidade de uso desse parâmetro na predição das propriedades de madeiras ou compostos de madeira.

O uso do $E_{m d}$ possibilitou a geração de modelos satisfatórios para determinação da resistência a $f_{c, 0}$ (Figura 5). Esse resultado está de acordo com os de diversos autores (JUNG, 1982; CHA; PEARSON, 1994; KRESTSCHMANN et al., 1993; KIMMEL; JANOWIAK, 1995), que têm comprovado a eficiência dos métodos não destrutivos para determinação das constantes elásticas de compostos estruturais de madeira.

No ensaio de $f_{c, 0}$, o $\mathrm{E}_{\mathrm{md}}$, obtido pelo método de ondas de tensão com propagação no sentido edgewise, foi o que obteve o maior $\mathrm{R}^{2}(0,70)$. Nogueira e Ballarin (2008), ao estudarem a qualificação mecânica da madeira de Eucalyprus sp. por diferentes métodos não destrutivos (ondas de tensão, ultrassom vibração transversal), envidenciaram que as melhores estimativas foram obtidas quando utilizadas ondas de tensão.

$\mathrm{O}$ ajuste de equação para estimar a resistência ao $f_{v g, 0}$ e $f_{v g, 90}$ não foi possível para o nível de confiança mínimo preestabelecido (95\%). Isso pode ser explicado pela variabilidade das amostras, já que estas tinham pequenas dimensões (amostras ensaiadas 


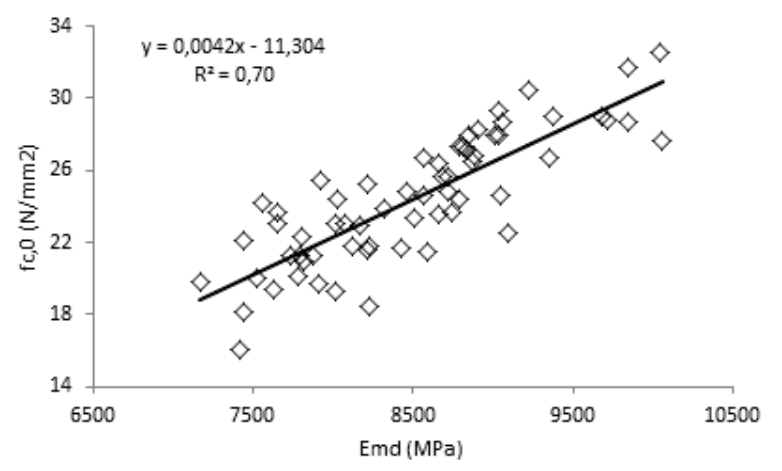

Figura 5 - Estimativa da resistência à compressão paralela às fibras $\left(f_{c \varrho}\right)$, em função do módulo de elasticidade dinâmico $\left(E_{m d}\right)$ obtido por meio de stress wave com propagação no sentido edgewise. ** Significativo a 0,01 .

Figure 5-Prediction of the resistance to parallel compression to fibers $\left(f_{c, 0}\right)$ as a function of thedynamic elasticity modulus $\left(E_{\text {mod }}\right)$ obtained by the stress wave propagating in the edgewise direction. **Significance at 0.01 .

destrutivamente) diante das que foram utilizadas nos ensaios não destrutivos. Especificamente no caso do $f_{v g}$, o outro aspecto que pode ter contribuído para esse resultado é o fato de ser o adesivo o principal componente que fornece a resistência nesse tipo de ensaio. Assim, para os fatores observados pelo Stress Wave $\left(E_{m d} \mathrm{e}\right.$ $V_{0}$ ) não foi possível estimar características relacionadas à qualidade da ligação e, consequentemente, com o emprego desses parâmetros também não foi possível gerar equações para predição da resistência nesse tipo de ensaio (UYSAL, 2005).

\section{CONCLUSÃO}

O método de avaliação não destrutiva, utilizando stress wave, pode ser utilizado na predição das propriedades físicas e mecânicas dos compostos LVL produzidos com paricá.

Para o inchamento residual e a resistência a esforços de cisalhamento, paralelos ou perpendiculares, o uso dessa ferramenta não possibilitou ajustes de modelos significativos.

O módulo de elasticidade dinâmico foi o parâmetro que melhor se adequou para estimar as propriedades dos LVL.

A velocidade de propagação das ondas não permitiu, na maioria dos casos, o ajuste de modelos estatisticamente significativos.
As amostras ensaiadas não destrutivamente, na posição edgewise, proporcionaram os modelos de melhores ajustes.

\section{AGRADECIMENTOS}

À Coordenação de Aperfeiçoamento de Pessoal de Nível Superior (CAPES), pela concessão da bolsa de doutorado; e ao Instituto Nacional de Ciência e Tecnologia de Madeiras da Amazônia (INCT/CNPq), pelo financiamento de partes dos estudos. Adicionalmente, ressalta-se a gratidão ao Centro de Pesquisa do Paricá (CPP) e às empresas Rio Concren ${ }^{\circledR}$ e Portil ${ }^{\circledR}$, pela concessão das lâminas.

\section{REFERÊNCIAS}

ALMEIDA, C. F. A. M. Uso de avaliação não destrutiva em vigotas de angelim vermelho. 2011. 43f. Monografia (Graduação em Engenharia Florestal) - Universidade de Brasília, Brasília, 2011.

\section{AMERICAN SOCIETY FOR TESTING AND}

MATERIALS - ASTM D 1037. Standard test methods of evaluating properties of wood-based fiber and particle panel materials. ASTM D 1037. Annual book of ASTM standards, 1999.

\section{AMERICAN SOCIETY FOR TESTING AND}

MATERIALS - ASTM D 5456. Standard specification for evaluation of structural composite lumber products. Annual book of ASTM standards, 2006.

\section{BORtoletto Júnior, G. Produção de lâminas, compensado e laminatedveneerlumber - LVL a partir da madeira de Pinus merkusii. 2006. 110f. Tese (Livre Docência) - Universidade de São Paulo/ ESALQ - Piracicaba, 2006.}

CHA, J. K.; PEARSON, R. G. Stress analysis and prediction in 3-layer laminated veneer lumber: response to crack and grain angle. Wood and Fiber Science, v.26, n.1, p.97-106, 1994.

DEL MENEZZI, C. H. S. Instabilidade dimensional da madeira e de seus subprodutos: causas, conseqüências e prevenção. Brasília, DF: Universidade de Brasilia, 2006. 37p. (Comunicações Técnicas Florestais, v. 8, n. 2).

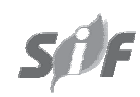

Revista Árvore, Viçosa-MG, v.38, n.6, p.1155-1163, 2014 
GABRIEL, M. S. C. Desempenho físicomecânico de painéis LVL de pinus tropicais da região de São Paulo. 2007. 157f. Tese (Doutorado em Agronomia - Energia na Agricultura) - Universidade Estadual Paulista, Botucatu, 2007.

HALABE, U.B.; BIDIGALU, G.M.; GANGARAO, H.V.S.; ROSS, R.J. Nondestructive evaluation of green wood using stress wave and transverse vibration techniques. Materials Evaluation, v.55, n.9, p.1013-1018, 1997.

JUNG, J. Properties of parallel laminated veneer from stress-wave-tested veneers. Forest Products Journal, v.32, n.7, p.30-35, 1982.

KIMMEL, J. D.; JANOWIAK, J. J. Red maple and yellow-poplar LVL from ultrasonically rated veneer. Forest Products Journal, v.45, n.7/ 8, p.54-58, 1995.

KRETSCHMANN, D.E.; MOODY, R.C.; PELLERIN, R.F.; BENDTSEN, B.A.; CAHILL, J.M.; MCALISTER, R.H. ; SHARP, D.W. Effect of various proportions of juvenile wood on laminated veneer lumber. USDA. Forest Service. Madison: FPL, 1993. 31 p. (ResearchPaper, FPL-521).

MATOS, J. L. M. Estudo sobre a produção de painéis estruturais de lâminas paralelas de Pinus taeda L. 1997. 117f. Tese (Doutorado em Ciências Florestais) -

Universidade Federal do Paraná, Curitiba, 1997.

MELO, R. R.; DEL MENEZZI, C. H. S. Influência da massa específica nas propriedades físicomecânicas de painéis aglomerados. Silva Lusitana, v. 18, n.1, p.59-73, 2010.

MELO, R. R.; DEL MENEZZI, C.H.S.; SOUZA, M.R.; STANGERLIN, D.M. Avaliação das Propriedades Físicas, Químicas, Mecânicas e de Superfície de Lâminas de Paricá

(Schizolobiumamazonicum Huber ex. Ducke).

Floresta e Ambiente, v.20, p.238-249, 2013.

MELO, R. R.; DEL MENEZZI, C.H.S. Inûuence of veneer thickness on the properties of LVL from Parica ' (Schizolobiumamazonicum) plantation trees. European Journal of Wood and Wood Products, v.72, n.2, p.191-198, 2014.
MORALES, E.A.M.; BERTOLINI, M.S.; NASCIMENTO, M.F.; LAHR, F.A.R.; BALLARIN, A.W. Study of brazilian commercial osb (oriented strand board) panels using stress wave. Wood Research, v.58, n.2, p.295-306, 2013.

NOGUEIRA, M.; BALLARIN, A. W. Efeito da idade na qualificação mecânica da madeira serrada de Eucalyptus sp. com uso de ensaios nãodestrutivos. Revista Energiana Agricultura, v.23, n.3, p.74-94, 2008.

PELLERIN, R. F.; ROSS, J. R. Nondestructive evaluation of wood. Madison: FPS, 2002. 210 .

ROSS, R.J.; ERICKSON, J.R.; BRASHAW, B.K.; WANG, X.; VERHEY, S.A.; FORSMAN, J.W.; PILON, C.L. Yield and ultrasonic modulus of elasticity of red maple veneer. Forest Products Journal, v.54, n.12, p.220-225, 2004

SANTOS, A. M. L. Avaliação teórica e experimental de vigas em “I” préfabricadas de madeira com flange de painéis de lâminas paralelas (LVL) e alma de painéis de partículas orientadas (OSB) e compensado. 2008. 94f. Dissertação (Mestrado em Ciências Florestais) - Universidade de Brasília, Brasília, 2008.

SHIMOYAMA, V. R. S. Estimativas de propriedades da madeira de Pinus taeda através do método nãodestrutivo de emissão de ondas de tensão, visando à geração de produtos de alto valor agregado. 2005. 115f. Tese (Doutorado em Engenharia Florestal) Universidade Federal do Paraná, Curitiba, 2005.

SOUZA, F. Propriedades mecânicas, físicas, biológicas e avaliação não destrutiva de painéis de lâminas paralelas (LVL) Confeccionados com Madeira de Pinus oocarpa e Pinus kesiya. 2009. 135f. Dissertação (Mestrado em Ciências Florestais) - Universidade de Brasília, Brasília, 2009.

SOUZA, F.; DEL MENEZZI, C.H.S.; BORTOLETTO JÚNIOR, G. Material properties and nondestructive evaluation of laminated veneer 
lumber (LVL) made from Pinusoocarpa and P. kesiya. European Journal of Wood and Wood Products, v.69, n.2, p.183-192, 2011.

STANGERLIN, D. M. et al. Propagação indireta e semidireta de ondas ultrassonoras na estimativa de propriedades mecânicas da madeira. Ciência da Madeira, v.2, n.2, p.85-95, 2011.

SUCHSLAND, O. Hygroscopic thickness swelling and related properties of selected commercial particleboards. Forest Products Journal, v.23, n.7, p.26-30, 1973.

TREVISAN, R. Variação radial da massa específica básica da madeira Eucalyptusgrandis W. Hill exMaiden. Floresta, v.38, n.3, p.553-559, 2008.
UYSAL, B. Bonding strength and dimensional stability of laminated veneer lumbers manufactured by using different adhesives after the steam test. International Journaul od Adhesion and Adhesives, v.25, n.5, p.395-403, 2005.

VALLANCE, D. B. Non-destructive evaluation of veneer using optical scanning and ultrasonic stress wave analysis systems. 2009. 279f. Tese (Doutorado em "Wood Science") - Oregon State University, Oregon, 2009.

XUE, B.; HU, Y. Nondestructive testing of mechanical properties of pine laminated veneer lumber. Advanced Materias Research, v.150/151, n.10, p.1435-1428, 2011. 
